\title{
DESVELANDO NUESTRA IDENTIDAD DIGITAL
}

\section{Linda Castañeda y Mar Camacho}
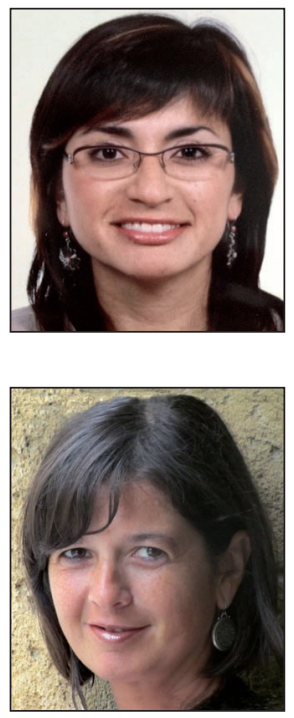

Linda Castañeda es pedagoga por la Universidad de Murcia (UM) y doctora en tecnología educativa por la Universitat de les Illes Balears. Profesora del Departamento de Didáctica y Organización Escolar de la Facultad de Educación de la UM, es miembro del Grupo de Investigación de Tecnología Educativa de esa universidad. Autora de diversos trabajos relacionados con la web 2.0, las redes sociales, los entornos personales de aprendizaje y en general el impacto de las tecnologías emergentes en los contextos de aprendizaje formales, no formales e informales.

$$
\begin{array}{r}
\text { Universidad de Murcia, Facultad de Educación } \\
\text { Campus de Espinardo. } 30100 \text { Murcia, España } \\
\text { lindacq@um.es }
\end{array}
$$

Mar Camacho es doctora en tecnología educativa. Docente e investigadora en el Departamento de Pedagogía de la Facultad de Educación de la Universitat Rovira i Virgili (Tarragona), es miembro desde 2001 del Grupo de Investigación de Tecnología Educativa de la misma universidad. Imparte clases y cursos en diferentes programas de master, doctorado y postgrado relacionados con la tecnología educativa. Es autora de diversas publicaciones sobre el uso de las TIC en los procesos de aprendizaje, el uso de la web 2.0 y sus herramientas y recursos para la docencia y sobre el uso y aplicación del mobile learning.

Univ. Rovira i Virgili, Fac. de Ciències de l'Educació i Psicologia, Dept. de Pedagogia Campus Sescelades. Ctra. de Valls, s/n. 43007 Tarragona, España mar.camacho@urv.cat

\section{Resumen}

Dado el nuevo concepto de identidad, consecuencia de los cambios tecnológicos y de comunicación actuales, se hace una aproximación crítica al concepto de identidad digital para desenmarañar su complejidad. Se presentan los resultados de una primera aproximación práctica, un estudio en el que se han analizado las percepciones de los alumnos de último año de universidad sobre su identidad digital, su nivel de preocupación en relación con la misma y la influencia de ésta en su presente y futuro profesional.

\section{Palabras clave}

Identidad digital, Comunicación, Educación, Redes sociales online, Web 2.0.

\section{Title: Revealing our digital identity}

\section{Abstract}

We critique the concept of digital identity and try to deal with all its complexity. In addition, we present findings from a first approach to this object of analysis, a study that explores higher education students' perceptions and concerns about their digital identity. Thus, this study focuses on digital identity as it is projected mainly on social networking sites (SNS) and the role they play in young people's lives.

\section{Keywords}

Digital identity, Communication, Education, Social networking, Social networks, Web 2.0.

Castañeda, Linda; Camacho, Mar. "Desvelando nuestra identidad digital”. El profesional de la información, 2012, julioagosto, v. 21, n. 4, pp. 354-360.

http://dx.doi.org/10.3145/epi.2012.jul.04

\section{Identidad digital y nuevos medios}

Tomaremos como punto de partida el papel de las tecnologías como mediadoras de la identidad (entendida como un concepto plural y dinámico), asumiendo que el concepto "identidad digital" se refiere a los aspectos de la tecnología digital como mediadora en la experiencia de la identidad construida por las personas y también condicionada por factores sociales. Con estos presupuestos, resultará muy relevante tratar de desentrañar cuáles son los cambios que 
están asociados presumiblemente con la identidad en estos tiempos digitalizados y cuáles las implicaciones de esos cambios a la hora de mejorar las posibilidades de nuestros procesos y modelos educativos.

Se hace una primera aproximación teórica al concepto de identidad digital, haciendo una revisión de los planteamientos más relevantes de la bibliografía científica existente, para luego estructurar una perspectiva teórica coherente que permita entender la complejidad del concepto y de las partes que lo configuran.

Uno de los objetivos es llamar la atención sobre cuestiones que consideramos críticas a la hora de pensar en el desarrollo integral de las personas en tanto que ciudadanos en un mundo de comunicación, de su trayectoria como aprendices permanentes y de sus relaciones sociales. Cuestiones que configuran los patrones básicos de relación y comunicación en los tiempos que corren y que deberían ocupar la atención de nuestros estudiantes -y de todos nosotros-en tanto que miembros activos de la sociedad, futuros profesionales y ciudadanos de pleno derecho.

\section{Ubicuidad, participación e intemporalidad en la web social: los detonantes de la identidad}

Si tenemos en cuenta que las tecnologías se han integrado de forma profunda y a la vez transversal en nuestra cotidianidad, es cada vez más evidente su rol como mediadoras de nuestra identidad y de nuestro aprendizaje, especialmente cuanto más invisibles se hacen para nuestros ojos (Weber; Mitchell, 2008; Weber; Dixon, 2007). Somos "residentes" en un mundo embebido de tecnologías (White; Le-Cornu, 2011).

Siguiendo el planteamiento de Tom-Tong et al. (2008, p. 1), en la mayoría de las experiencias de lo que llamábamos hasta hace poco computer-mediated-communication (CMC) las cuestiones relativas a la relación entre actividades de comunicación y los juicios interpersonales han adquirido una enorme importancia, siendo estas cuestiones decisivas a la hora de entender la naturaleza de nuestras experiencias sociales y culturales -especialmente las de los más jóvenes-y la forma cómo éstas afectan a nuestra identidad (Buckingham, 2008).

Actualmente, la preocupación por la identidad se intensifica de manera espectacular promovida por el aumento exponencial del uso de internet en general, la web social y las redes sociales en internet, en particular (Castañeda et al., 2011). No en vano hablamos de un tipo de herramientas que, sólo en España, han aumentado su uso de un $45 \%$ de la población total en 2008 a un 94\% en 2011 (Cocktail Análisis, 2012) y que concentran el uso de una mayoría extraordinaria de la población, especialmente los jóvenes.

En este marco, y desde una perspectiva teórica, muchas de las aproximaciones conciben la identidad más como un proceso que como un concepto estático. La identidad es algo que no puede ser conseguido de una vez y para siempre, sino que es fluida y está permanentemente abierta a la negociación, a la vez que se encuentra limitada por diversos factores (Weber; Mitchell, 2008).
Hoy en día, uno de los factores que más afecta a la identidad es el mundo de relaciones y actividad propios del contexto digital (hablamos incluso de identidad digital). Da igual nuestra edad o nuestro contexto social y cultural, la comunicación mediada por la tecnología y los procesos que se llevan a cabo a través de ella afectan a todos, al menos en lo que llamamos el mundo conectado (Wheeler, 2009).

Hemos pasado a una sociedad que, por defecto y gracias a los avances tecnológicos, recuerda para siempre

En este sentido, Mayer-Schoenberger (2007) recuerda que hemos pasado de una sociedad que por defecto "olvidaba", a una sociedad que, por defecto y gracias a los avances tecnológicos "recuerda para siempre". Este cambio ha impactado en nuestra habilidad para participar de forma libre y esa nueva realidad -la de que todo será recordado para la posterioridad-tiene efectos importantes en la forma en la que nos relacionamos, que aún no han sido suficientemente explorados.

Es interesante el trabajo de Valkenburg y Peter (2008) en el que investigan los efectos que tiene la experimentación de la identidad online por parte de adolescentes holandeses en el desarrollo de su competencia social. Así, los adolescentes que más a menudo experimentaron con su identidad en internet también se comunicaron online y con más frecuencia con personas de diferentes edades y antecedentes culturales. Esta comunicación, a su vez, tuvo un efecto positivo sobre la competencia social del adolescente, pero no afectó a su autoconcepto. Según el estudio, la competencia social de los adolescentes se benefició considerablemente de estos experimentos de identidad online, de lo que se deduce que internet les proporciona oportunidades para conectar con otra gente. Al hacerlo, los jóvenes parecen usar a estas personas como cajas de resonancia para explorar su identidad y practicar, con éxito, sus habilidades de sociales.

En esta misma línea, Baumgartner et al. (2010) reflejan el impacto -a veces nocivo- que las actividades llevadas a cabo por los jóvenes en internet pueden tener en su desarrollo. Así, pasar mucho tiempo en la Red puede llevarles a transferir actividades tradicionalmente llevadas a cabo fuera de línea y afectar sustancialmente el desarrollo adolescente. Es por este motivo que actividades online que pueden ser potencialmente peligrosas para los adolescentes deben investigarse más a fondo.

No podemos obviarlo, los efectos a los que nos referimos tienen un impacto evidente en nuestra capacidad para construir y manejar nuestra identidad e incluyen, como no, la parte digital de la misma que a su vez, como puntualizan los autores, tiene múltiples aristas y no sólo está configurada por lo que publicamos sobre nosotros mismos en la Web.

\section{La compleja estructura de la identidad digital}

Es precisamente en la combinación entre sociedad, cultura y tecnologías digitales donde radican hoy las mayores 
posibilidades para el aprendizaje y el enriquecimiento de la identidad de las personas (Goldman et al. 2008). Como consecuencia, es necesario hacer conscientes a todos los ciudadanos, de la forma en la que se desarrollan ambos aprendizaje e identidad-, especialmente en un mundo cada vez más sofisticado desde un punto de vista tecnológico (Notley, 2009).

\section{La identidad es algo fluido y dinámico, y se ha revelado además, como un concep- to complejo y multidimensional, (Childs)}

La identidad es algo fluido y dinámico, y se ha revelado además, como un concepto complejo y multidimensional (Childs, 2010; Peachey; Childs, 2011). Podríamos asimilar este concepto con el denominado por Manders-Huits (2010, p. 48)) "identidad nominal", es decir el conjunto de atributos asignados a una persona por la sociedad, atributos que necesitan estar fijados -aunque sea de forma temporal- para que esa persona pueda ser identificada y re-identificada de forma consistente. Esa identidad nominal se organiza en torno a hechos y cosas relacionadas con esa persona en un rol o situación concreto (Childs, 2010).

Coincidimos con la opinión de diversos autores (Tom-Tong et al., 2008; Greeanhow et al., 2009; Childs, 2010; Peachey; Childs, 2011; Manders-Huits, 2010; Notley, 2010 que afirman que existe una variada cantidad de factores y hechos de nuestra "vida online" que influyen poderosamente (lo que llamamos la identidad digital), algunos dependientes de nosotros mismos -la parte personal de nuestra identidad-y otros que escapan de nuestro control directo y dependen de otras personas -la parte más social de dicha identidad-.

Pero veámoslo por partes.

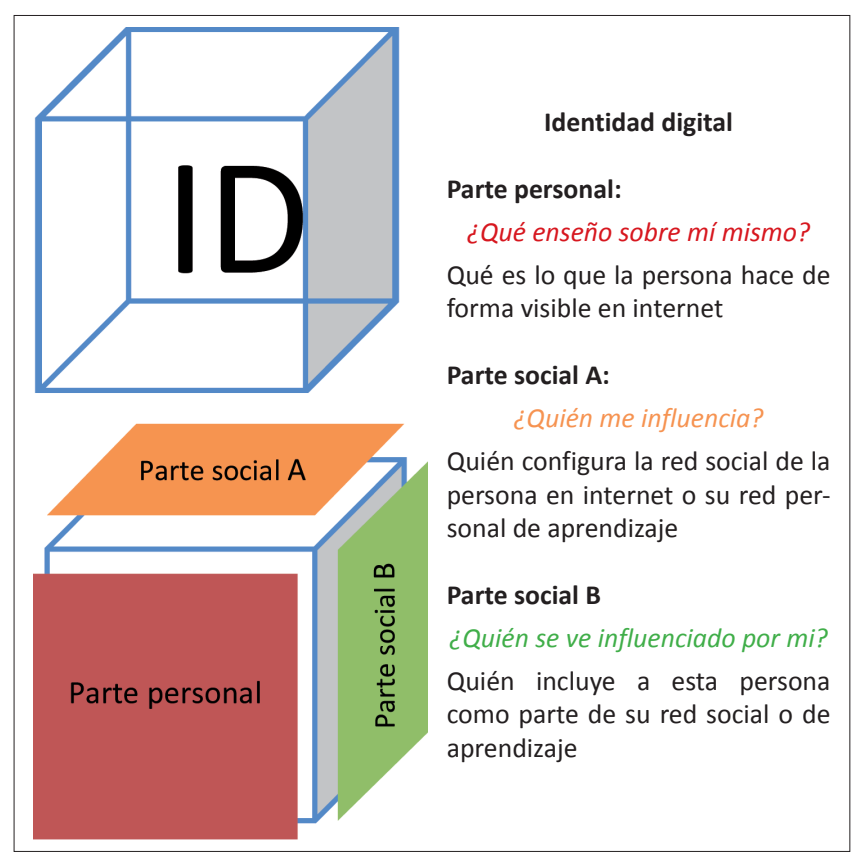

Figura 1. Identidad digital
En lo que se refiere a nuestra identidad, nuestra visión de nosotros mismos en la Red y lo que hacemos en ella "es sólo parte de la pintura completa" (Peachey; Childs, 2011, p. 31). La "parte personal" de la identidad digital de una persona es proyectada por lo que esa persona hace en la Red: qué dice, cómo lo dice, el lenguaje que usa, sus temas de interés y un largo etcétera que configuramos básicamente cada vez que hacemos click en el ordenador, y que reforzamos cuando damos nuestro voto positivo o negativo a una cosa u otra. Es el reflejo de nuestra imagen en el espejo de la Red.

Pero, al igual que sucede de forma presencial, nuestra identidad en la Red tiene una "parte social" que se configura en torno a dos formas básicas que no dependen de nosotros mismos, sino de nuestras relaciones:

- En primer lugar tenemos a aquellos que nos influencian formando parte de nuestra red social de contactos o de nuestra red social de aprendizaje (amigos, contactos, personas a las que seguimos, etc.). Son aquellos a los que oímos, retuiteamos, con los que hablamos, aquellos a los que comentamos su contenido y por los que mostramos interés en la Red. De ellos, de sus comportamientos, de sus gustos, aficiones y de la información que comparten con nosotros y de cómo esa información circula entre nosotros, los demás sacan conclusiones también sobre nuestra propia personalidad, gustos, etc. Sí, aquello de "dime con quién andas..." también tiene un reflejo en internet.

- Por otra parte, existe un grupo de personas (más o menos grande en la medida de nuestro impacto personal o profesional) en el que nos reflejamos. Son las personas que se ven afectadas o influenciadas por nosotros (nuestros seguidores, aquellos que comentan nuestro contenido, etc.), sean o no sean nuestros amigos -o tengamos mutuo contacto con ellos- y estén o no de acuerdo con nosotros. Interpretan nuestras palabras, se hacen eco de ellas y les sirven como canal de distribución. En algunos casos son sus palabras y no las nuestras las que tienen un impacto directo en la Red y las que ayudan a que nuestra imagen quede reflejada de una forma $u$ otra.

\section{Construyendo identidad aun sin ser conscientes}

Las presencia de los estudiantes universitarios, sus relaciones y su capacidad para formar parte de comunidades de práctica, de aprendizaje, de desarrollo profesional, así como para gestionar su propia imagen, han sido poco exploradas en el contexto Red, al menos de momento (Moorman; Bowker, 2011; Castañeda; Camacho, 2010), aun cuando es en este período -el de los estudios universitarios- cuando este tipo de relaciones y la identidad que se forja suelen ser determinantes para el futuro profesional de las personas (Goode; 2010).

Lo sepan o no, como afirma Shareski (2010), los estudiantes están continuamente expresando, compartiendo, transmitiendo, formalizando, presentando y desarrollado su propia identidad personal, presencial y en red. Sin embargo, las implicaciones que sus acciones "cotidianas" tienen sobre su propia persona y sobre su identidad no están suficientemente estudiadas, siendo muy probable que ni ellos ni 
nosotros seamos muy conscientes de cómo puede afectar toda su actividad en la construcción de su identidad digital. Son especialmente sensibles las actuaciones realizadas en las herramientas que ligan nuestras acciones a nuestro nombre de forma evidente (Zhao et al., 2008) como es el caso del software social y especialmente el de las redes sociales online (para una distinción entre ambos términos, v. Castañeda, 2010).

Para comprobar este aspecto hemos hecho un estudio exploratorio con una muestra de 164 estudiantes de último curso de la titulación de magisterio en el curso 2010-2011 (132 de ellos, mujeres). El objetivo ha sido conocer hasta qué punto existe entre ellos conciencia sobre su identidad digital, las partes que la configuran, el rol que asume en ella su actividad en las redes sociales y el impacto de internet en sus vidas. Por ello el estudio se realizó entre los que durante dicho curso académico cursaban la asignatura específica sobre tecnología educativa.

La mayoría de los estudiantes dijo haber oído hablar de casi todas las herramientas de la web social, aunque tienen un perfil de bajo uso. Los que dicen usar las diferentes tecnologías han asumido el papel de lurker (Preece et al., 2004), es decir, utilizan la herramienta para buscar y ver el contenido de otras personas, pero no publican nada. Sólo en el caso de las redes sociales online (RRSS), el porcentaje de participantes que declara asumir el papel de lurker es sólo del $2 \%$ y afirman que habitualmente aportan contenido.

Los estudiantes están preocupados por la imagen de sí mismos que proyectan en internet pero sólo de una forma social inmediata

\subsection{Conciencia sobre la parte personal de la propia identidad}

La mayoría de nuestros estudiantes utiliza Tuenti $(82,4 \%)$ y Facebook $(73,9 \%)$ como redes sociales. Y casi todos usaron Tuenti antes que Facebook (en adelante $F B$ ).
La gran mayoría de encuestados (84\%) afirma ser consciente de las opciones de privacidad que les ofrecen las redes sociales, y suelen tener su perfil abierto a los amigos de sus amigos, aunque ninguno de ellos lo tenga completamente oculto.

Sorprende que el $12,6 \%$ de los usuarios de Tuenti no sabe o no se preocupa por conocer la cantidad de fotos de ellos mismos que hay publicadas y más del $5,7 \%$ pasa por alto este dato en el caso de $F B$. Pero resulta aún más interesante que el $6,7 \%$ de los usuarios de Tuenti no sabe o no le preocupa saber la cantidad de fotos que él mismo ha publicado, mientras que un $4,1 \%$ dice ignorar esta misma información en relación con $F B$.

Cuando se les preguntó qué creen que refleja su comportamiento en la Red (lo que dicen y hacen), la mayoría de ellos tiene la impresión de ser visto por los demás como inteligente $(134$, el $81,7 \%)$, interesante $(116,70,7 \%$ ) y trabajador $(124,70,6 \%)$, y no creen que otros puedan verles como egoístas $(133,81,1 \%)$, manipuladores $(120,73,2 \%)$, huraños $(114,69,5 \%)$ o tristes $(108,65,9 \%)$.

\subsection{Conciencia de la parte social de la identidad}

La mayoría de los encuestados no creen que los demás ejerzan mucha influencia sobre ellos (90 de 164, 54,9\%), si bien un 20,1\% reconoce que está muy influenciado por el contenido y los comentarios publicados por sus amigos. Sin embargo sólo $46(28,1 \%)$ aseguran tener bastante influencia sobre sus amigos.

Cuando se les preguntó sobre otra parte de su imagen online: la opinión que puede hacerse otra gente sobre ellos basándose en las personas a las que siguen, las apreciaciones son similares a las que se referían a sus propias acciones; piensan que son percibidos como inteligentes $(118,72,0 \%)$, interesantes $(115,70,1 \%)$, trabajadores $(92,56,1 \%)$ y profundos $(85,51,8 \%)$, mientras que no creen que otros los puedan consideran egoístas $(133,81,1 \%)$, tristes $(121,73,8 \%)$, huraños $(121,73,8 \%)$ o manipuladores $(107,65,2 \%)$.

Por último, al ser consultados sobre la opinión de la gente basada en los comentarios que otros hacen sobre los con-

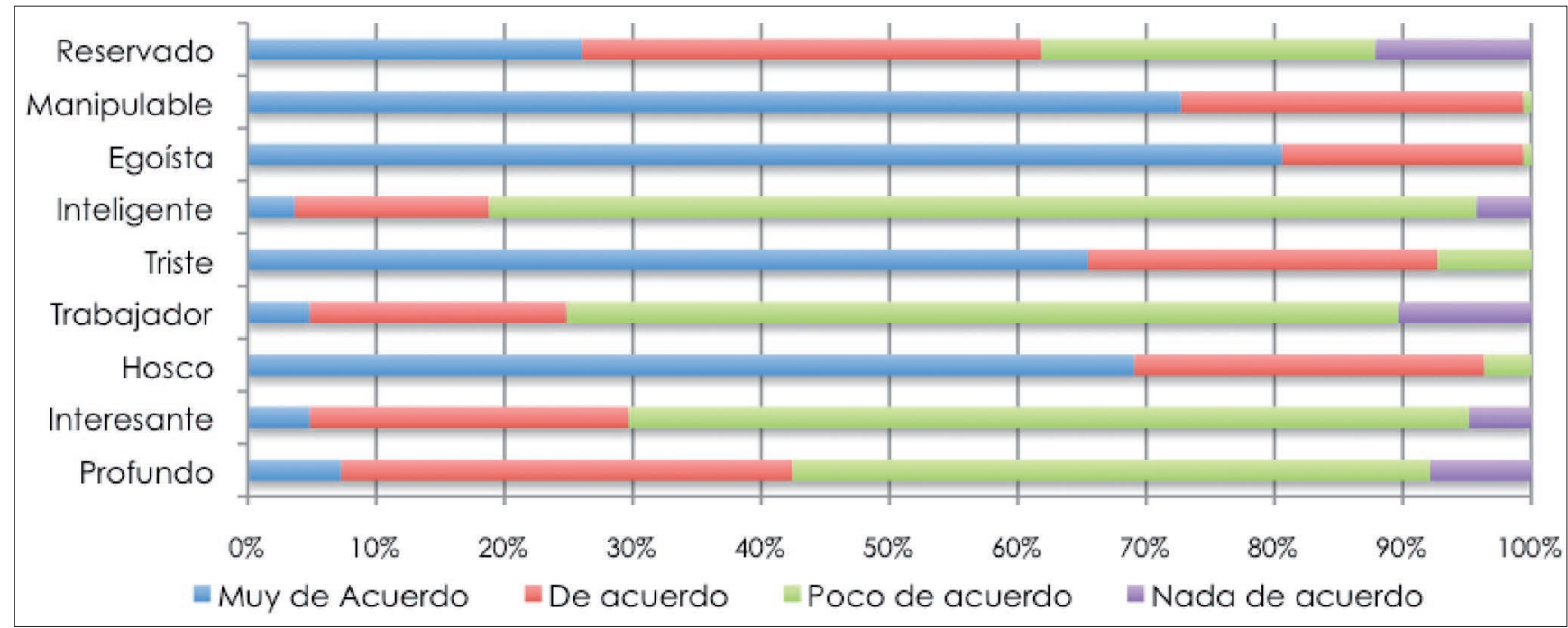

Figura 2. ¿Cómo te ven los demás de acuerdo con lo que ven de ti en internet? 


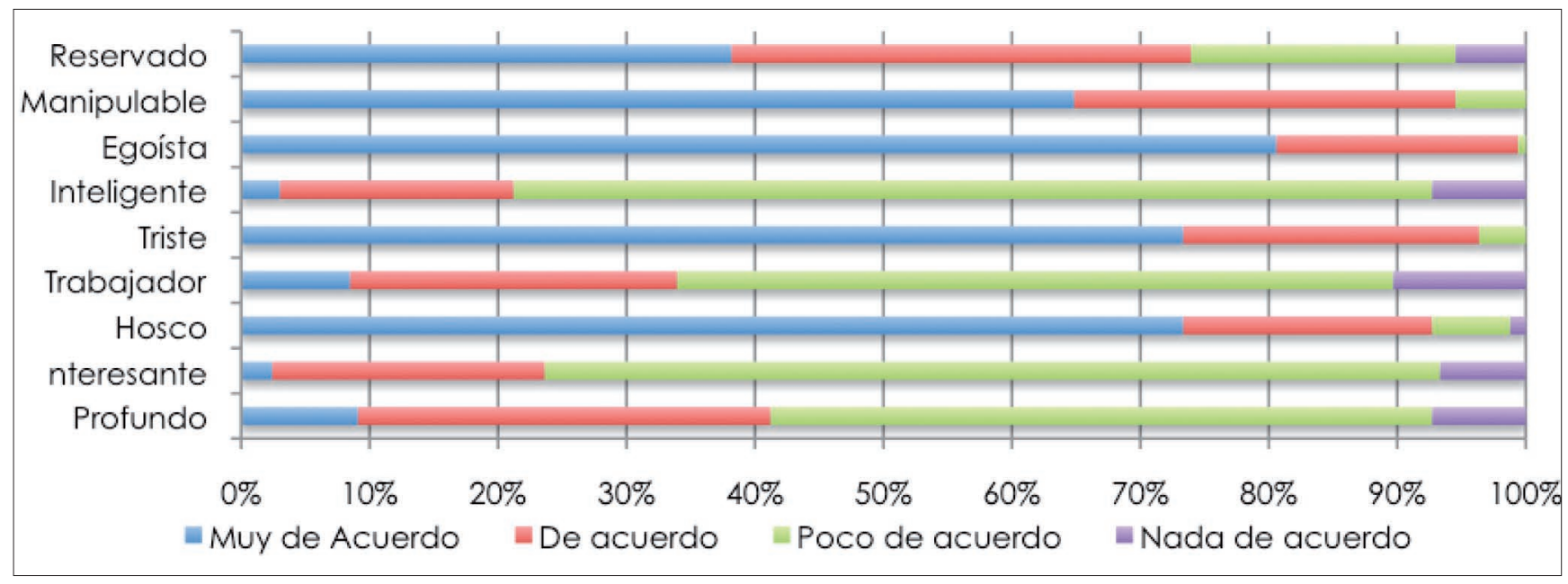

Figura 3. ¿Cómo te ven los demás de acuerdo con las personas a las que sigues/reflejas en internet?

tenidos que ellos publican en las RRSS, tampoco parece haber muchas diferencias: consideran que pueden ser vistos por los demás como inteligentes $(118,72,0 \%)$, interesantes $(108,65,9 \%)$, trabajadores $(92,56,1 \%)$ y profundos $(80$, $48,8 \%)$ y se niegan a la idea de que los demás puedan verlos como egoístas $(136,82,9 \%)$ tristes $(121,73,8 \%)$, huraños $(121,73,8 \%)$ o manipuladores $(113,79,0 \%)$.

\section{3. ¿Y el impacto de su identidad digital en sus vi- das?}

Casi un $94 \%$ coincide en que a veces, casi siempre o siempre, publica contenidos sin pensar en su impacto en la Red y un $80 \%$ de ellos reconoce haber borrado contenido después de haberlo publicado. El $80 \%$ dice no tener conciencia de que otras personas -aparte de sus amigos- pueden ver sus actualizaciones y comentarios, ni se ha planteado situaciones hipotéticas en las que pueda suceder, aunque un $23 \%$ declara que no le preocupa que así sea.

En cuanto a sus preocupaciones, el $75 \%$ está preocupado por la imagen que sus amigos puedan hacerse sobre ellos basándose en su comportamiento en la Red. Pero un $26 \%$ coincide en afirmar que no le preocupa en absoluto el impacto de esa imagen en su futura carrera.

Por último, les pedimos que calificaran de 1 a 10 cuánto se parece su "yo" reflejado en las redes a su "yo" real, y la nota media de sus opiniones -7,6- no deja lugar a demasiadas dudas, sí creen que son ellos mismos en las redes sociales.

Conocer la forma en que la identidad se crea y configura nos ayudará a comprender nuestros experiencias sociales y culturales

\section{Algunas conclusiones y pensamientos sueltos}

Creemos firmemente que conocer la forma en que la identidad se crea y configura nos llevará a una mejor comprensión de la naturaleza de nuestros experiencias sociales y culturales y puede ayudarnos a mejorar de forma consciente nuestros procesos de aprendizaje y relación con el entorno.

Los resultados de la exploración que ofrecemos muestran que aunque los estudiantes son conscientes de que su identidad puede ser explicada por muchos factores externos, esto no parece que les preocupe en gran medida.

Los estudiantes participantes están preocupados por la imagen de sí mismos que proyectan a través de internet pero sólo de una forma social inmediata. Uno de cada 4 estudiantes no está preocupado en absoluto por el impacto

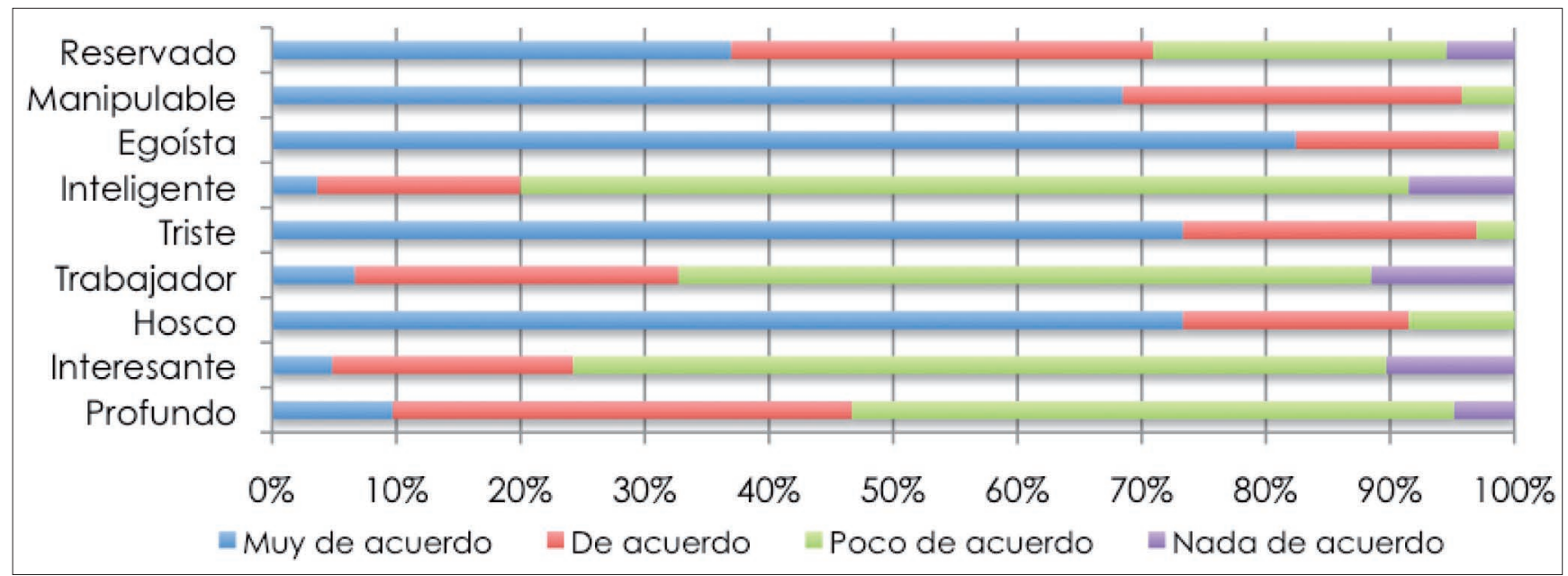

Figura 4. ¿Cómo te ven los demás por lo que otras personas dicen de ti en internet? 
de su actividad digital en su futuro profesional, hecho que debe tenerse en cuenta a la hora de abordar procesos de empleabilidad no sólo en el ámbito de la educación, sino en cualquiera de los campos profesionales.

Es interesante ver que los estudiantes universitarios no conocen el impacto que la vida digital puede tener sobre su vida diaria. La necesidad de capacitación y orientación específica sobre éstos y otros hechos relacionados con el uso y la gestión de su propia identidad parece esencial, sobre todo teniendo en cuenta las implicaciones que esta gestión encarnan en el desarrollo de sus futuras carreras profesionales.

De cara al futuro sería muy interesante ampliar nuestra visión sobre ésta y otras cuestiones de forma sistemática. Creemos que uno de los caminos evidentes para explorar se refiere a cuáles son las motivaciones de los usuarios a la hora de ceder información sobre su persona a la Red, así como para publicar uno u otro tipo de información en una $u$ otra herramienta social. El estudio más cercano de los comportamientos de los estudiantes y sus dinámicas en estos entornos de relación podría revelarnos datos interesantes para un mayor conocimiento de las vías posibles de desarrollo de la identidad digital en el marco de la educación.

Los estudiantes universitarios no conocen el impacto que la vida digital puede tener sobre su vida diaria

Como sucede en la vida cotidiana, los jóvenes aprenden de manera informal sobre las posibilidades y las preocupaciones que tienen que tener en cuenta, aunque algunos de ellos no sean conscientes ni de las repercusiones ni de dicho aprendizaje. Sin embargo, está claro que entender las nociones implícitas de la identidad nos puede ayudar a facilitar el diseño de nuevas y mejores estrategias educativas (aplicables en contextos formales y no formales) en el ámbito de la gestión de esa identidad (Manders-Huits, 2010) y creemos realmente que dicho ámbito tiene que constituir una parte fundamental de la educación básica de los futuros ciudadanos, y por lo tanto una parte vital de su formación profesional.

\section{Bibliografía}

Baumgartner, Susanne E.; Valkenburg, Patti M.; Peter, Jochen. "Assessing causality in the relationship between adolescents' risky sexual online behavior and their perceptions of this behavior". Journal of youth and adolescence, 2010, v. 39, n. 10, pp. 1226-1239.

http://www.ncbi.nlm.nih.gov/pmc/articles/PMC2917006 http://dx.doi.org/10.1007/s10964-010-9512-y

Buckingham, David. "Introducing identity". En: Buckingham, David (ed.). Youth, identity, and digital Media. The John D. and Catherine T. MacArthur Foundation series on digital media and learning. Cambridge, MA: The MIT Press, 2008, pp. 1-24.

http://mitpress.mit.edu/books/chapters/0262294273 chap1.pdf
Castañeda, Linda. "Aprendizaje con redes sociales". Tejidos educativos para los nuevos entornos. Sevilla: MAD, 2010. ISBN: 97884677636192

Castañeda, Linda; González, Víctor; Serrano, José-Luis. "Dónde habitan los jóvenes: precisiones sobre un mundo de redes sociales". En: Martínez, Francisco; Solano, Isabel. Comunicación y relaciones sociales de los jóvenes en la Red. Alicante: Marfil, 2011. ISBN: 9788426815545 http://digitum.um.es/xmlui/bitstream/10201/25353/1/ castaneda_gonzalez_serrano.pdf

Castañeda, Linda; Camacho, Mar. "A strange in the mirror? Students perceptions about their digital identity". En: Bastiaens, Theo; Ebner, Martin (eds.). Procs of the World conf on educational multimedia, hypermedia and telecommunications, 2011, pp. 3275-3280. Chesapeake, VA: AACE.

Childs, Mark. "A conceptual framework for mediated environments". Educational research, 2010, v. 52, n. 2, pp. 197213.

http://dx.doi.org/10.1080/00131881.2010.482738

Cocktail Análisis. Observatorio de Redes Sociales. IV Oleada. Informe público de resultados, 2012, abril.

http://www.slideshare.net/TCAnalysis/4-oleada-observato rio-de-redes-sociales

Goldman, Shelley; Booker, Angela; McDermott, Meghan. "Mixing the digital, social, and cultural: learning, identity, and agency in youth participation". En: Buckingham, David. (ed.). Youth, identity, and digital media. The John D. and Catherine T. MacArthur Foundation series on digital media and learning. Cambridge, MA: The MIT Press, 2008, pp. 185206.

http://www.mitpressjournals.org/doi/pdfplus/10.1162/ dmal.9780262524834.185

Greenhow, Christine; Robelia, Beth. "Old communication, new literacies: social network sites as social learning resources. Journal of computer-mediated communication, 2009, v. 14, pp. $1130-1161$.

http://dx.doi.org/10.1111/j.1083-6101.2009.01484.x

Goode, Joanna. "The digital identity divide: how technology knowledge impacts college students". New media \& society, 2010 , v. 12 , n. 3, pp. 497-513.

http://dx.doi.org/10.1177/1461444809343560

Leiva-Aguilera, Javier. Gestión de la reputación online. Colección EPI-UOC, n. 7. Barcelona: Editorial UOC, 2012, 98 pp. ISBN: 9788497889902

Manders-Huits, Noëmi. "Practical versus moral identities in identity management". Ethics and information technology, 2010, v. 12, n. 1, pp. 43-55.

http://es.scribd.com/doc/80657344/5/Practical-versusMoral-Identities-in-Identity-Management http://dx.doi.org/10.1007/s10676-010-9216-8

Mayer-Schönberger, Viktor. "Useful void: the art of forgetting in the age of ubiquitous computing". KSG faculty research working paper series, RWP07-022, 2007, April. http://www.vmsweb.net/attachments/pdf/Useful_Void.pdf

Moorman, Jessica; Bowker, Anne. "The university Facebook experience: the role of social networking on the qual- 
ity of interpersonal relationship". The American Association of Behavioral and Social Sciences journal (AABSS), 2011, v. 15, pp. 1-23.

http://aabss.org/Journal2011/04MoormanFinal.pdf

Notley, Tanya. "Young people, online networks, and social inclusion". Journal of computer-mediated communication, 2009, v. 14, n. 4.

http://eprints.qut.edu.au/17278

http://dx.doi.org/10.1111/j.1083-6101.2009.01487.x

Peachey, Anna; Childs, Mark. Reinventing ourselves: contemporary concepts of identity in virtual worlds. London, New York: Springer, 2011. ISBN: 9780857293602

http://www.springerlink.com/content/978-0-85729-361-

9\#section $=920026 \&$ page $=1 \&$ locus $=0$

http://dx.doi.org/10.1007/978-0-85729-361-9

Preece, Jenny; Nonnecke, Blair; Andrews, Dorine. "The top five reasons for lurking: improving community experiences for everyone". Computers in human behavior, 2004, v. 20, n. 2, pp. 201-223.

http://dx.doi.org/10.1016/j.chb.2003.10.015

Shareski, Dean. Understanding you digital identity, 2010. http://www.slideshare.net/shareski/identity-managementproject

Tom-Tong, Stephanie; Van-Der-Heide, Brandon; Langwell, Lindsey; Walther, Joseph B. "Too much of a good thing? The relationship between number of friends and interpersonal impressions on Facebook". Journal of computer-mediated communication, 2008, v. 13, pp. 531-549.

http://campus.albion.edu/keithkaplan/files/2010/11/Num beroffriends.pdf

http://dx.doi.org/10.1111/j.1083-6101.2008.00409.x
Valkenburg, Patti M.; Peter, Jochen. “Adolescents' identity experiments on the internet: consequences for social competence and self-concept unity". Communication research, 2008 , v. 35, n. 2, pp. 208-231. http://dx.doi.org/10.1177/0093650207313164

Weber, Sandra; Dixon, Shanly (eds). Growing up online: children and technologies. New York: Palgrave, 2007. ISBN: 9780230620018

Weber, Sandra; Mitchell, Claudia. "Imaging, keyboarding, and posting identities: young people and new media technologies". Youth, identity, and digital media. The John D. and Catherine T. MacArthur Foundation series on digital media and learning. Cambridge, MA: The MIT Press, 2008, pp. 25-47.

http://www.mitpressjournals.org/doi/pdfplus/10.1162/ dmal.9780262524834.025

Wheeler, Steve. Multiple identities, 2010.

http://steve-wheeler.blogspot.com/2010/12/multiple-iden tities.html

White, David S.; Le-Cornu, Allison. "Visitors and residents: a new typology for online engagement". First Monday, 2011, Sept., v. 16, n. 9.

http://firstmonday.org/htbin/cgiwrap/bin/ojs/index.php/ $\mathrm{fm} /$ article/view/3171/3049

Zhao, Shanyang; Grasmuck, Sherri; Martin, Jason. "Identity construction on Facebook: digital empowerment in anchored relationships". Computers in human behavior, 2008, v. 24, n. 5 , pp. $1816-1836$.

http://astro.temple.edu/ bzhao001/Identity\%20Constructi on\%20on\%20Facebook.pdf

http://dx.doi.org/10.1016/j.chb.2008.02.012

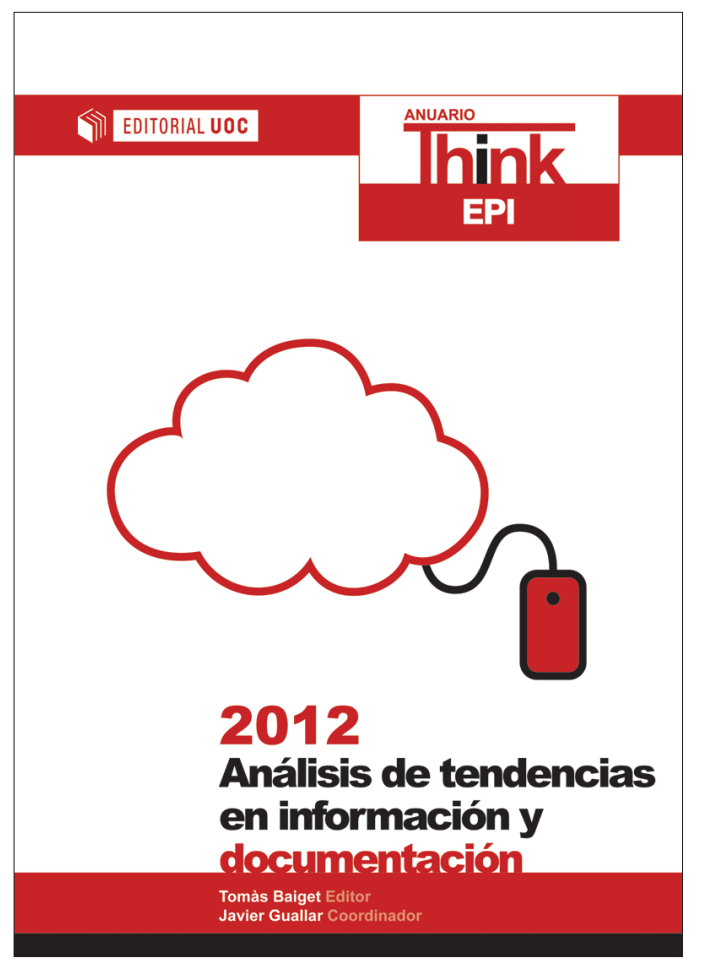

\title{
Ya ha salido el nuevo
}

\section{Anuario ThinkEPI 2012}

\author{
Información y adquisición en:
}

http://www.thinkepi.net/anuario-
thinkepi/anuario-thinkepi-2012

y

http://www.editorialuoc.cat/
anuariothinkepi2012-p-985.htm/ 